\title{
TAU Mutations are not a Predominant Cause of Frontotemporal Dementia in Canadian Patients
}

\author{
Anastasia Levchenko, Yves Robitaille, Michael J. Strong, Guy A. Rouleau
}

\begin{abstract}
Objective: Frontotemporal dementia is a neurodegenerative disease affecting mostly the frontal and/or temporal lobes, with neuronal loss and intraneuronal and/or intraglial inclusions composed of hyperphosphorylated microtubule-associated protein tau and ubiquitin. Missense and splice site mutations in the TAU gene have been identified in approximately $15 \%$ of all frontotemporal dementia cases. In this study, we evaluated the involvement of mutations in the TAU gene in development of frontotemporal dementia phenotype in patients of French or English Canadian origins. Methods: Fourteen patients with frontotemporal dementia phenotype and 98 normal controls were recruited for the study. The TAU gene was screened by sequencing and denaturing high performance liquid chromatography. Results: No mutations, except some new polymorphisms, were detected in the $T A U$ gene of these patients. One polymorphism, however, may play a role in pathogenesis. Conclusion: Our results agree with previous work suggesting that mutations in this gene are not a frequent cause of the frontotemporal dementia phenotype in Canadian patients.
\end{abstract}

RÉSUMÉ: Les mutations du gène TAU ne sont pas une cause fréquente de démence fronto-temporale chez les patients canadiens. Objectif: La démence fronto-temporale est une maladie neurodégénérative touchant surtout les lobes frontaux et/ou temporaux, avec perte neuronale et intraneuronale et/ou inclusions intragliales composées de la forme hyperphosphorylée de la protéine tau et d'ubiquitine associées aux microtubules. Des mutations dans le gène $T A U$ ont été identifiées chez à peu près $15 \%$ de tous les cas de démence fronto-temporale. Nous avons évalué l'implication des mutations du gène $T A U$ dans le développement de la démence fronto-temporale chez des patients d'origine canadienne française et anglaise. Méthodes: Quatorze patients atteints de démence fronto-temporale et 95 témoins normaux ont été recrutés pour participer à l'étude. Le gène TAU a été séquencé et soumis à la chromatographie haute performance en phase liquide dénaturante pour détecter la présence de mutations. Résultats: Aucune mutation, sauf de nouveaux polymorphismes, n'a été détectée dans le gène $T A U$ de ces patients. Cependant, un polymorphisme pourrait jouer un rôle dans la pathogenèse. Conclusion: Nos résultats sont conformes aux travaux antérieurs suggérant que les mutations dans ce gène ne sont par une cause fréquente de démence fronto-temporale chez les patients canadiens.

Can. J. Neurol. Sci. 2004; 31: 363-367

Frontotemporal dementia (FTD) is a group of clinically heterogeneous neurodegenerative diseases that include Frontotemporal Dementia with Parkinsonism linked to chromosome 17 (FTDP17), progressive supranuclear palsy, corticobasal degeneration, Pick's disease, and FTD associated with motor neuron disease/amyotrophic lateral sclerosis. All these diseases present similar neuropathological findings, the most striking of which are neuronal loss and gliosis predominantly in frontal and/or temporal cortices, with subcortical nuclei, brain stem nuclei and spinal cord anterior and/or posterior horns also frequently affected. Moreover, one of the hallmarks of this group of diseases are intracytoplasmic inclusions called neurofibrillary tangles (NFT). They are immunopositive for the protein tau in a hyperphosphorylated state and are found in neurons as well as in glial cells. The role of NFT in neuronal toxicity and loss in FTD remains controversial. ${ }^{1,2}$ Moreover, intracytoplasmic inclusions found in some FTD cases stain only for ubiquitin. ${ }^{3}$

Tau is a microtubule-associated protein that binds to tubulin monomers, which constitute the microtubules and promote

From the Center for Research in Neuroscience, McGill University Health Center Research Institute,(AL, GAR); Department of Pathology and Cellular Biology, University of Montreal, and Pathology Department, Ste-Justine Hospital, (YR) Montreal, Quebec, Department of Clinical Neurological Sciences, London Health

Sciences Centre, University Campus, London, Ontario, (MJS); Canada. ReCEIVED APRIL 01, 2003. ACCEPTEDIN FINALFORM JANUARY 15, 2004. Reprint requests to: Guy A. Rouleau, Center for Research in Neuroscience, McGill University Health Center Research Institute, 1650 Cedar Avenue, Montreal, Quebec, Canada, H3G 1A4 
microtubule polymerization and stability. Tau is specific to axons in which the microtubules play an important role in cell traffic and maintenance of the shape of the axon. ${ }^{1}$ Glia (astrocytes and oligodendrocytes) also express this gene but the consequence of tau aggregates in these cells is still unclear. ${ }^{1,2}$

Tau is expressed as six isoforms, produced by alternative splicing of different exons. One half of the isoforms contain the exon 10, while the other half do not. This exon encodes for one of the four microtubule-binding domains of the protein, which are composed of repetitive amino acid motifs. Three of the isoforms contain four such domains, while the other three contain three domains. The two types of the isoforms are designated $4 \mathrm{R}$ and $3 \mathrm{R}$. The central nervous system (CNS) ratio of $4 \mathrm{R}$ to $3 \mathrm{R}$ is close to one, but slight variations are detected in different parts of the CNS. ${ }^{1-4}$

Baker et $\mathrm{al}^{5}$ identified a series of polymorphisms spread through the MAPT gene. These polymorphisms constitute two extended haplotypes that cover the gene. In a total of approximately 200 unrelated Caucasian individuals, no recombination between the two haplotypes was detected. This suggests that the establishment of the two haplotypes was an ancient event and that either recombination was suppressed in this region, or recombination was selected against. The more common haplotype is designated H1 (also A), and the less common one - $\mathrm{H} 2$ (also B). . $^{5-7}$

About $40 \%$ of FTD cases are familial and in approximately $40 \%$ of familial cases mutations in the TAU gene were detected. ${ }^{8}$ More than 20 different mutations segregating with the disease phenotype have been reported. ${ }^{1,2}$ The mutations are almost

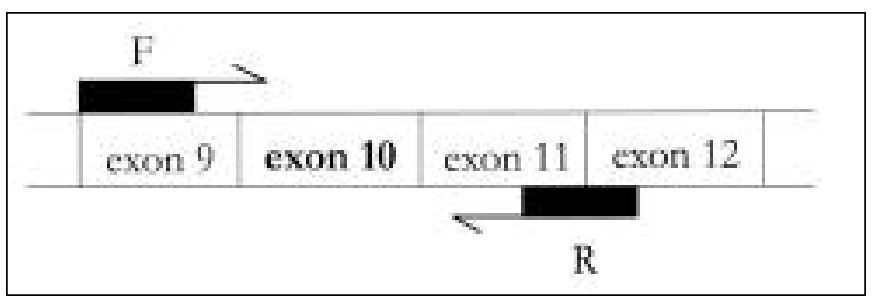

Figure 1: Position of the two primers on the cDNA of TAU. $F$ : forward primer; $R$ : reverse primer. exclusively grouped in and around exons 9,10 , and 12 , which encode for the four microtubule-binding domains, and exon $13 .{ }^{1}$ One half of those are missense mutations apparently reducing tau's capacity to bind to microtubules and stabilize them, whereas the other half, clustered in and around exon 10, seem to affect the alternative splicing of this exon and result in this exon being more frequently retained in TAU transcripts. ${ }^{1,2,4,8-10}$ This apparently changes the normal 3R/4R isoform ratio of tau, which affects the microtubule-binding capacity of tau. As a consequence, all mutations seem to result in an increase of unbound tau in the cytoplasm of neurons (and/or glia), ${ }^{1,11,12}$ which is thought to be the primary event in the formation of the intraneuronal and/or intraglial tau aggregates. ${ }^{13}$

\section{METHODS AND MATERIALS}

\section{Subjects}

All samples were obtained after approval of the Ethics Committee of the Douglas Hospital, Québec, Canada. A total of 10 unrelated patients of French Canadian origin and four unrelated patients of English Canadian origin, with different subtypes of FTD, were recruited for the study (Table 1). Patients were either from Québec or Ontario, Canada. Six patients had a familial history (i.e. presence of at least one other affected family member). Final diagnosis of dementia-amyotrophic lateral sclerosis (ALS) was established clinically in four patients, two of whom had family history of ALS and one, of FTD-ALS. In the other 10 patients, the clinical diagnosis was that of a dementia (one patient with dementia-ALS). Their final diagnosis of FTD was established on neuropathology by an expert neuropathologist (Y.R.) using the Lund and Manchester Groups criteria for FTD ${ }^{14}$ (Table 2). The neuropathological examination revealed: two cases of classical FTD, one of which had a primary progressive aphasia; two cases of progressive supranuclear palsy, one of which had a family history; one case of corticobasal degeneration; and five less well-defined cases for which Alzheimer's type dementia was definitely excluded. These were: two unspecified dementias (both with family history), one dementing syndrome with severe ataxia and dysartria, and two cases of cerebral senile changes with mental confusion, one of

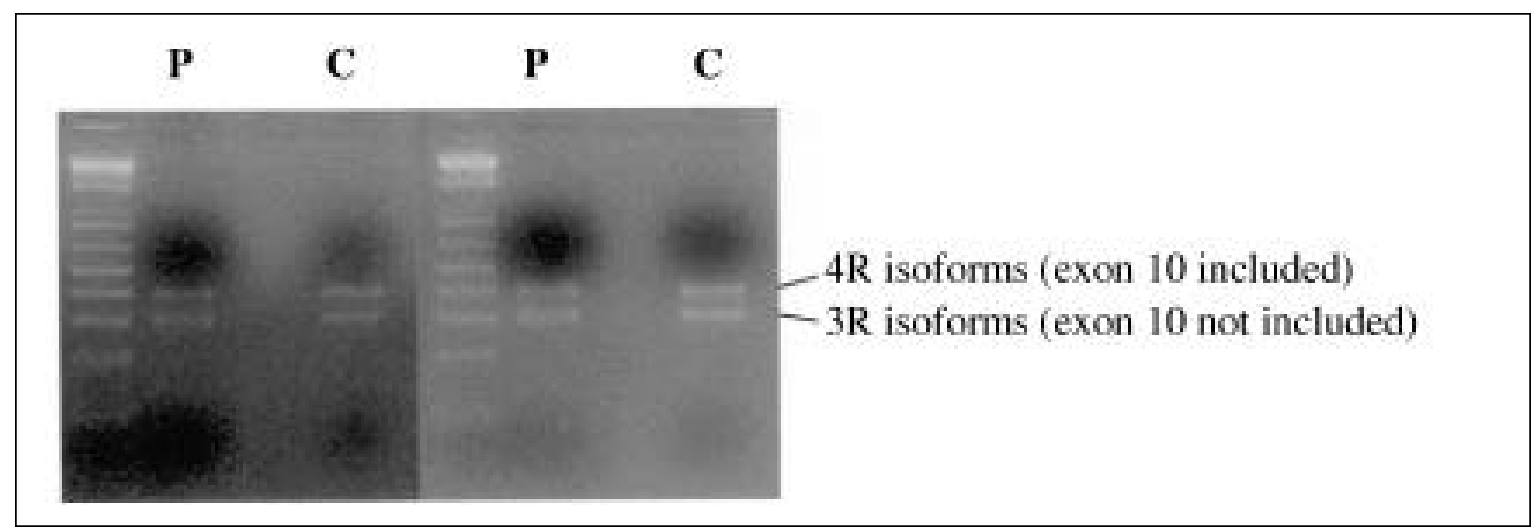

Figure 2: Results of the quantitative PCR of cDNA of tau on a $2 \%$ agarose gel. $P$ : patient; $C$ : normal control 


\section{Table 1: List of screened subjects}

\begin{tabular}{llll}
\hline Case & Final Diagnosis & Family History & Ethnic Origin \\
FTD & neuropathological & negative & French Canadian \\
FTD-aphasia & neuropathological & negative & French Canadian \\
FTD-ALS & clinical & positive (FTD-ALS) & English Canadian \\
PSP & neuropathological & positive (PSP) & French Canadian \\
PSP & neuropathological & negative & French Canadian \\
CBD & neuropathological & negative & French Canadian \\
Unspecified dementia & neuropathological & positive (dementia) & French Canadian \\
Unspecified dementia & neuropathological & positive (dementia) & French Canadian \\
Unspecified dementia & neuropathological & negative & French Canadian \\
dementia-ALS & neuropathological & negative & French Canadian \\
dementia-ALS & clinical & negative & English Canadian \\
dementia-ALS & clinical & positive (ALS) & English Canadian \\
dementia-ALS & clinical & positive (ALS) & English Canadian \\
Unspecified dementia-ataxia/dysarthria & neuropathological & negative & French Canadian
\end{tabular}

FTD, frontotemporal dementia; ALS, amyotrophic lateral sclerosis; PSP, progressive supranuclear palsy; CBD, corticobasal degeneration

\section{Table 2: Criteria for neuropathological diagnosis of FTD subtypes}

\section{FTD subtype Most prominant neuropathological lesions}

FTDP17 Pick cells (ballooned neurons); gliosis; severe neuronal loss and NFT in hippocampus, frontal and temporal lobes, and substantia nigra; degeneration in cranial nerves and spinal cord anterior horns

PiD Pick cells; gliosis; severe neuronal loss and NFTin form of Pick bodies in hippocampus, singular and parahippocampal gyri, and frontal and temporal lobes

PSP Pick cells; gliosis; severe neuronal loss and NFT in hippocampus, frontal and temporal lobes, globus pallidus, substantia nigra, tegmentum, and locus coeruleus

CBD Pick cells; gliosis; severe neuronal loss and NFTin hippocampus, singular and parahippocampal gyri, frontal and temporal lobes, globus pallidus, substantia nigra, tegmentum, and locus coeruleus

FTD-MND/ALS Pick cells; gliosis; severe neuronal loss and NFT in hippocampus, frontal and temporal lobes, and substantia nigra; degeneration in cranial nerves and spinal cord anterior horns; ubiquitin inclusions in dentate gyrus of the hippocampus

FTD, frontotemporal dementia; FTDP17, frontotemporal dementia with Parkinsonism linked to chromosome 17; PiD, Pick's disease; PSP, progressive supranuclear palsy; CBD, corticobasal degeneration; FTD-MND/ALS, FTD associated with motor neuron disease/amyotrophic lateral sclerosis; NFT, neurofibrillary tangles

\section{Table 3: Polymorphisms of the TAU gene}

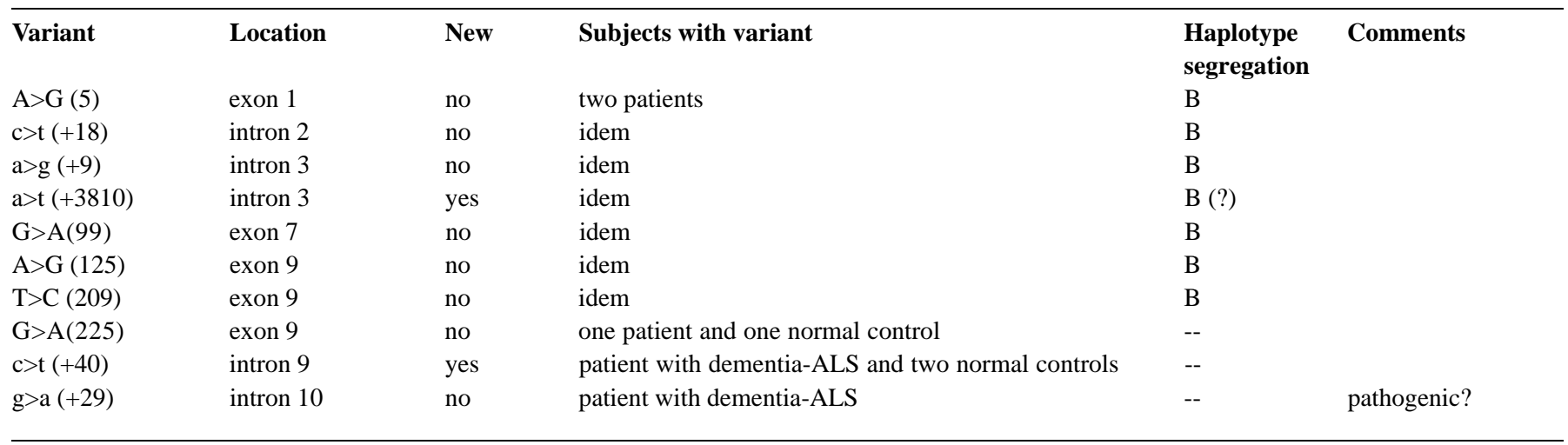

$\mathrm{A}=$ adenine $; \mathrm{C}=$ cytosine $; \mathrm{T}=$ thymine $\mathrm{G}=$ guanine 
which was the case clinically diagnosed with ALS. Ninety-eight normal controls from the same age group and geographic area were recruited for the study. For the 10 patients examined neuropathologically as well as three normal controls, DNA was obtained from frozen brain tissue (cerebellum) using standard methods. For the other normal controls, as well as the other four patients, DNA was extracted from whole blood using standard procedures.

\section{Genetic studies}

Only exons expressed in the CNS (TAU genomic sequence at $\mathrm{http} /$ /genome.UCSC.edu), i.e. exons 1, 2, 3, 4, 5, 7, 9, 10, 11, 12, and 13 were screened. Primers and polymerase chain reaction (PCR) amplification conditions were described elsewhere. ${ }^{15}$ Each of the amplicons - exons and their neighbouring intron regions - were analyzed by denaturing high performance liquid chromatography on the WAVE DNAFragment Analysis System (Transgenomics, Omaha, USA). This method allows the detection of heteroduplexes (annealing of two DNAstrands with one to several base pair mismatch because of the presence of different alleles) in a PCR product. It is based on the fact that the melting temperature for a heteroduplex is lower than that for a homoduplex (two perfectly annealed DNA strands). So, at a given melting temperature, the heteroduplex will denaturate earlier in time. The presence of DNA in elution is then detected by the apparatus, and the presence of the heteroduplex will be registered as a separate peak (also called variant) of the UV light absorption. Samples with faint and strong variants detected on denaturing high performance liquid chromatography were subsequently sequenced using the big dye terminator chemistry on an ABI 3700 sequencer (Applied Biosystems) using the same primers as for the PCR. Sequence alignments were performed using SeqMan II software from DNASTAR Inc. For direct sequencing, PCR products were purified from an agarose gel using Qiax II Gel Extraction Kit (Qiagen) and the Thermo Sequenase Radiolabeled Terminator Cycle Sequencing Kit ${ }^{33} \mathrm{P}-$ labeled) from USB was used. Results of direct sequencing were read on the BIOMax film from Kodak.

An RT-PCR was performed to evaluate the $4 \mathrm{R} / 3 \mathrm{R}$ tau ratios. The positions of the primers used are presented on the Figure 1 and their sequences are: CCCGCACCCCGTCCCTTCC (forward primer) and TTACTTCCACCTGGCCACCTCCTG (reverse primer). The two following quantitative PCRs were done with reduced numbers of cycles, 23 and 27 , so to avoid erasing differences between the two isoforms by an overamplification.

\section{RESULTS}

A total of 10 variants were detected in this study (Table 3). Some of the variants have already been reported in literature as $T A U$ polymorphisms also present in the healthy population (the $255 \mathrm{G}$ to A variant in exon 9, also found in one normal control in this study, and the $\mathrm{G}$ to A substitution situated at +29 of the intron $10^{8,15}$ ). Two of the variants were not previously reported and thus were considered as new polymorphisms. The $\mathrm{C}$ to $\mathrm{T}$ substitution 40 base pairs inside the intron 9 was also present in two normal controls, and for that reason the possibility that it is a pathogenic mutation was excluded. The A to T substitution 3,810 base pairs inside the intron 3 is located 94 bases pairs before the exon 4 .
The possibility that it is a pathogenic mutation was ruled out based on two reasons: (1) it does not disrupt the splicing consensus motives for either the exon 3 or 4 ; (2) while the $\mathrm{A}$ is conserved in the mouse, it is not in the rat, which makes it less likely to be functionally important.

Six sequence variations, that are part of the haplotype $\mathrm{H} 2$, were seen only in two of the patients. ${ }^{6}$ These patients were therefore carriers of the haplotype $\mathrm{H} 2$. They were also the same ones that presented the A to $\mathrm{T}$ substitution 3,810 base pairs inside the intron 3, which makes it possible that this variant is a previously undetected part of the haplotype $\mathrm{H} 2$.

However, the $\mathrm{G}$ to A change at +34 of the intron 11 , which normally would be found on the haplotype $\mathrm{H} 2,{ }^{6}$ was not found in these two subjects. They both had the genotype G/G (usually found on the haplotype $\mathrm{H} 1$ ).

The $\mathrm{G}$ to $\mathrm{A}$ substitution is located 29 base pairs inside the intron 10 and the $\mathrm{G}$ is conserved in both mouse and rat. To determine if the presence of the A allele affects the alternative splicing of this exon, a quantitative PCR for the cDNA region flanking the exon 10 (Figure 1) was performed in order to estimate the relative abundances of the $4 R$ and $3 R$ isoforms of tau. The quantitative PCR did not show any difference in abundances between the $4 \mathrm{R}$ and $3 \mathrm{R}$ isoformes in the patient with this variant (Figure 2).

\section{DiscuSsion}

It has become clear that, despite the importance of the discovery of TAU mutations as a cause of the FTD phenotype, mutations in this gene do not explain the majority of cases of FTD (approximately 15\%) and not even the majority of familial cases (approximately 40\%). ${ }^{15,16}$ Our results support this view by showing a lack of disease phenotype-associated mutations in the $T A U$ gene in a cohort of French Canadian and English Canadian patients with the FTD phenotype. Indeed, it is likely that this phenotype in our patients is caused by different and/or yet unknown molecular pathological processes. The identification of additional genes responsible for FTD is critical in order to understand the full spectrum of pathological phenomena evolving in the CNS of FTD patients. A number of candidate genes have been proposed, including cytoplasmic kinases such as Cdk5 and MAPT, ${ }^{17,18}$ and/or phosphatases, ${ }^{17}$ considering that tau is found in a hyperphosphorylated state in the NFT/inclusions. In addition, candidate chromosome loci include a 9q21-22 locus linked to the FTD-ALS phenotype ${ }^{19}$ and a 3p11.1-q11.2 locus (OMIM \#600795) linked to an unspecified dementia phenotype.

This study allowed discovery of two new polymorphisms in the TAU gene, without apparent role in the disease. One of the new variants, the $A$ to $T$ in the intron 3 , was not detected in normal controls. However, it is not conserved in the rat and it does not disrupt any known splice site; it was also detected in the same two individuals that were carriers of the $\mathrm{H} 2$ haplotype. It is thus possible that this polymorphism is a part of the $\mathrm{H} 2$ haplotype and that it may simply have been missed by previous studies.

The absence of one previously published element of haplotype $\mathrm{H} 2$, the $\mathrm{A}$ at the position 34 in the intron 11 ( $\mathrm{G}$ in case of the haplotype $\mathrm{H} 1$ ), in the two patients clearly segregating the $\mathrm{H} 2$ haplotype, may be explained in two different ways. There 
may have occurred a recombination between the two haplotypes, which previously were believed to never recombine..$^{5-7}$ This is possible given that the variant is at the end of the haplotype. Secondly, because the two patients are both of the French Canadian origin, the A allele may have changed to a $\mathrm{G}$ de novo in a common $\mathrm{H} 2$ haplotype bearing ancestor.

The $\mathrm{G}$ to A substitution situated at +29 in intron 10 was previously described as a polymorphism, because it does not change the 4R/3R tau isoform ratios, ${ }^{8}$ which was also confirmed in our study (Figure 2). It was however present only in affected subjects in our study; and it is also conserved in both the mouse and the rat. This variant might therefore be pathogenic by some yet undetermined mechanisms and may even be causing the FTD-ALS phenotype, since the patient with the variant had a clinical diagnosis of ALS complicated with dementia. Further studies are needed to resolve this question.

\section{ACKNOWLedGements}

We thank Danielle Cécyre, the coordinator at the Brain Bank of the Research Center of the Douglas Hospital, Québec, for providing samples for this study; Sandra Laurent for her contribution to the DNA variant analysis; and we thank to numerous students and technical assistants of Dr Rouleau's Neurogenetics Laboratory for their help in course of this project.

Anastasia Levchenko is supported by the Fonds de Recherche en Santé du Québec and Guy A. Rouleau is supported by the Canadian Institutes Of Health Research.

\section{REFERENCES}

1. Garcia ML, Cleveland DW. Going new places using an old MAP: tau, microtubules and human neurodegenerative disease. Curr Opin Cell Biol 2001; 13: 41-48.

2. Goedert $\mathrm{M}$. The significance of tau and $\alpha$-synuclein inclusions in neurodegenerative diseases. Curr Opin Genet Dev 2001; 11: 343351.

3. Rosso SM, Kamphorst W, de Graaf B, et al. Familial frontotemporal dementia with ubiquitin-positive inclusions is linked to chromosome 17q21-22. Brain 2001; 124: 1948-1957.

4. Hong M, Zhukareva V, Vogelsberg-Ragaglia V, et al. Mutationspecific functional impairments in distinct tau isoforms of hereditary FTDP-17. Science 1998; 282: 1914-1917.

5. Baker M, Litvan I, Houlden H, et al. Association of an extended haplotype in the tau gene with progressive supranuclear palsy. Hum Molec Genet 1999; 8: 711-715.

6. Ingelson $\mathrm{M}$, Fabre $\mathrm{SF}$, Lilius $\mathrm{L}$, et al. Increased risk for frontotemporal dementia through interaction between tau polymorphisms and apolipoprotein E $\varepsilon 4$. NeuroReport 2001; 12 : 905-909.

7. Houlden H, Baker M, Morris HR, et al. Corticobasal degeneration and progressive supranuclear palsy share a common haplotype. Neurology 2001; 56: 1702-1706.

8. D'Souza I, Poorkaj P, Hong M, et al. Missense and silent tau gene mutations cause frontotemporal dementia with parkinsonismchromosome 17 type, by affecting multiple alternative RNA splicing regulatory elements. Proc Natl Acad Sci USA1999; 96: 5598-5603.

9. Poorkaj P, D'Souza I, Bird TD, Schellenberg GD. Tau mutations: genetics and pathogenetic mechanisms. In: Lee VM-Y, Trojanowski JQ, Buee L, Christen Y, (Eds). Fatal Attractions: Protein Aggregates in Neurodegenerative Disorders. Berlin, Germany: Springer-Verlag Heidelberg, 2000: 53-64.

10. Varani L, Hasegawa M, Spillantini MG, et al. Structure of tau exon 10 slicing regulatory element RNA and destabilization by mutations of frontotemeporal dementia and parkinsonism linked to chromosome 17. Proc Natl Acad Sci USA 1999; 96: 82298234.

11. Hutton M. Missence and splice site mutations in tau associated with FTDP-17: multiple pathogenic mechanisms. Neurology 2001; 56: S21-S25.

12. Goedert M, Spillantini MG. Tauopathies and a-synucleopathies. In: Lee VM-Y, Trojanowski JQ, Buee L, Christen Y, (Eds). Fatal Attractions: Protein Aggregates in Neurodegenerative Disorders. Berlin, Germany: Springer-Verlag Heidelberg, 2000: 65-75.

13. Von Bergen M, Barghorn S, Li L, et al. Mutations of tau protein in frontotemporal dementia promote aggregation of paired helical filaments by enhancing local $\beta$-structure. J Biol Chem 2001; 276: 48165-48174.

14. Brun A, Englund B, Gustafson L, et al. (The Lund and Manchester Groups) Clinical and neuropathological criteria for frontotemporal dementia. J Neurol Neurosurg Psychiatry 1994; $57: 416-418$.

15. Rizzu P, van Swieten JC, Joosse M, et al. High prevalence of mutations in the microtubule-associated protein tau in a population study of frontotemporal dementia in the Netherlands. Am J Hum Genet 1999; 64: 414-421.

16. Morris HR, Khan MN, Janssen JC, et al. The genetic and pathological classification of familial frontotemporal dementia. Arch Neurol 2001; 58: 1813-1816.

17. Drewes G, Ebneth A, Preuss U, Mandelkow EM, Mandelkow E. MARK, a novel family of protein kinases that phosphorylate microtubule-associated proteins and trigger microtubule disruption. Cell 1997; 89: 297-308.

18. Iwatsubo T, Hasegawa M, Ihara Y. Neuronal and glial tau-positive inclusions in diverse neurologic diseases share common phosphorylation characteristics. Acta Neuropathol 1994; 88: 129-136.

19. Hosler BA, Siddique T, Sapp PC, et al. Linkage of familial Amyotrophic Lateral Sclerosis with Frontotemporal Dementia to chromosome 9q21-22. JAMA2000; 284: 1664-1669. 